Solomon, M.C. et al. (2014). In vivo effects of eurycoma longifolia jack (tongkat ali) extract on reproductive functions in the rat. Adrologia, 46(4): 339-48

\title{
Provision of Syndromic Treatment of Sexually Transmitted Infections by Community Pharmacists: A Potentially Underutilized HIV Prevention Strategy
}

Kim Ward, Nadine Butler, Pierre Mugabo, Jeffrey Klausner, Willi Mcfarland, Sanny Chen and Sandra Schwarcz,

\begin{abstract}
Background: Sexually transmitted infections (STIs) are known risk factors for HIV infection.

Goal: The goal of this study was to assess the current and potential future role that community pharmacists in Western Cape, South Africa play in the treatment of STIs.

Study Design: A cross-sectional survey of community pharmacists in the Western Cape region of South Africa. A face-to-face interview that ascertained experience with requests from patients for STI treatment, current STI treatment practices, and willingness to provide syndromic STI treatment was administered to head pharmacists.

Results: Ninety pharmacies were selected and 85 (94\%) of the head pharmacists participated; 55 from an urban area and 30 from a rural area. Pharmacists reported a median of 40 urban clients and 25 rural clients who sought STI treatment from community pharmacists. When provided with a hypothetical clinical situation, $13 \%$ of urban and $17 \%$ of rural pharmacists identified the correct medication for male urethral discharge, $8 \%$ of urban pharmacists and none of the rural pharmacists identified correct treatment for genital ulcers, and none of the pharmacists identified the correct medication for vaginal discharge. Fifty-three percent of pharmacists in urban regions and $47 \%$ of pharmacists in rural regions expressed willingness to provide syndromic STI treatment. Independent predictors of willingness to provide syndromic treatment were knowledge of the link between HIV transmission and STIs (adjusted odds ratio [OR]: 13.78; 95\% CI: 2.69, 70.66), past experience prescribing syndromic STI treatment (OR: 11.1; 95\% CI: 1.14, 108.6), and male gender (OR: 4.38; 95\% CI: 1.15 ,

16.7).

Conclusions: Pharmacists are frequently called upon to provide STI treatment but have limited knowledge of correct treatment recommendations. Training pharmacists to provide syndromic STI treatment may be one strategy to reduce STI morbidity and HIV transmission.
\end{abstract}

South Africa has one of the world's most severe HIV-1 epidemics: an estimated 4.7 million South Africans are infected with HIV, including 21.1\% of adults aged 15 to 49 years. ${ }^{1}$ Along with HIV, sexually transmitted infections (STIs) are highly prevalent in South Africa, with approximately 11 million new cases treated each year. Because STIs 
are known to increase the risk of transmitting HIV infection,3,4 the South African Department of Health developed an HIV/AIDS/STI strategic plan for 2000 to 2005, which calls for expanded efforts to treat and control STIs. One strategy for the control of STIs in developing countries is the use of syndromic treatment, which any healthcare worker may be trained to use. The plan specifies the need to ensure effective syndromic management of STIs in both the private and public sector.

The majority of South Africans rely on publicly provided healthcare services, which are inadequately funded and staffed. This may explain the anecdotal reports suggesting that patients seek and receive treatment for STIs from pharmacists, despite legal restrictions to this practice (Medicine and Related Substances Control Amendment Act of 1997), a phenomenon that is also reported in other developing countries such as Ghana and Peru. Thus, pharmacists may represent an alternative source of care for the treatment of STIs in a setting in which the healthcare system is overburdened. To understand the current and potential role that pharmacists play in the treatment of STIs, we conducted a survey of community pharmacists in urban and rural areas of the Western Cape region of South Africa. Rigorous HIV prevention strategies are desperately needed in the Western Cape, where STI incidence is high and HIV prevalence is still relatively low.

\section{Methods}

A list of all community pharmacies located in the Western Cape was obtained from the South African Pharmacy Council. Pharmacies were stratified into those located in the Cape Metropole, an urban area (population density 1,125.74/ $/ \mathrm{km}^{2}$ ), and those located in the rural area outside of the Cape Metropole (population density $11.39 / \mathrm{km}^{2}$ ). A simple random sample of pharmacies was selected from each stratum, proportional in size to the number pharmacies in each region.

The owner or manager of selected pharmacies was contacted by telephone by one of the study staff and informed of the study purpose and procedures. Only lead pharmacists, all of whom hold a bachelor of pharmacy degree or higher, were interviewed because discussions regarding use of prescription medications only occur between the clients and lead pharmacists or, in rare situations, a nurse based in the pharmacy. An appointment was made to conduct the face-to-face interview with those pharmacists who agreed to participate. Signed consent to participate was obtained in person immediately before conducting the interview.

Participating pharmacists were administered a structured interview that addressed the following key areas: (1) the characteristics of their pharmacies and clientele; (2) their knowledge, attitudes, and practices regarding STI management; and (3) their perceived obstacles to providing syndromic STI management. The Committees on Human Research of the University of California San Francisco and the University of Western Cape reviewed and approved this study.

\section{Analysis}

The chi-square test was used to identify statistically significant proportional differences between the characteristics of the pharmacies and the differences in the knowledge, 
attitudes, and practices regarding syndromic STI treatment between the pharmacists who worked in urban and rural pharmacies. Wilcoxon rank sum test was used to compare differences in medians.

Pharmacists were asked about their willingness to provide each individual step in the syndromic treatment of STIs. For the purposes of analysis, a pharmacist was defined as willing to provide syndromic STI treatment if he or she was willing to perform history taking, counseling for STI/HIV, partner notification, and follow-up, plus at least two of the following steps: visual or physical examination of genitals, prescribing medications, and phlebotomy for syphilis serology. We used this definition of willingness to provide syndromic treatment because these elements are required in the national STI syndromic treatment protocols.

Logistic regression was performed to measure the association between the pharmacists' willingness to provide syndromic treatment for STI and several predictor variables. The variables evaluated were the socioeconomic status of the clientele of the pharmacies, the location of the pharmacy (urban or rural), the gender of the pharmacist, the presence of a nurse at the pharmacy, the pharmacist's perception of the need for syndromic STI treatment, having received requests for dispensing medication for the treatment of vaginal discharge, genital ulcers, and urethral discharge from clients, and knowledge of the existence of syndromic STI treatment protocols and Department of Health STI management guide.

Pharmacists' knowledge of STI treatment was gauged from their responses to three hypothetical case studies: a male patient with genital ulcers, a male patient with urethral discharge, and a patient with vaginal discharge. The responses were assessed against a standard for the correct management of STI6 and defined as "selected effective combination of therapies" if the selected treatment was consistent with these standards, and as "selected partially effective therapies" if at least one effective medication was selected. Other responses were not considered to be effective.

For each of the clinical scenarios, the pharmacist was asked if he or she would "definitely," "maybe," or "not necessarily" refer the patient to a medical doctor, prescribe medication, and inform the client of HIV/AIDS risks. A response of definitely or maybe was considered to be affirmative. Pharmacists were classified as knowledgeable of the clinical and epidemiologic link between STI and HIV when HIV/AIDS risks were consistently mentioned in the three case studies. Among pharmacists who stated that they would provide medications for any of the clinical scenarios, the proportion that provided correct treatment (i.e., those who selected the correct combination of antibiotics as defined in national protocols) and partially correct treatment (selected at least one recommended antibiotic) was determined.

Results

Of the 406 community pharmacies located in the Western Cape, 90 (22\%) were sampled and 85 (94\%) agreed to be interviewed. Participating pharmacists from urban regions were more likely to be male than were pharmacists from rural regions $(P<0.001$, Table1). Pharmacies in both regions had fairly extensive hours of operation. Clientele 
represented diverse levels of income as described by the pharmacists, and this diversity did not differ between urban and rural pharmacies. Pharmacists reported receiving requests for medications without a doctor's prescription on a frequent basis, including requests for treatment of STI; urban pharmacists reported significantly more requests for STI treatment in the previous 4 weeks than did rural pharmacists.

In general, pharmacists regarded their role in the management of STI as underutilized, and the majority also strongly or slightly agreed that there is a need for STI treatment services to be provided in community pharmacies (Table 2). In both regions, a large proportion of the pharmacists indicated willingness to participate in syndromic treatment of STI. More than $90 \%$ of pharmacists from both regions were willing to take a history, counsel clients on prevention of STI and HIV, conduct partner-notification counseling, and follow up with the patient. Although all of the pharmacists in urban regions were willing to prescribe STI medication, fewer (87\%) pharmacists in rural regions were willing to do so $(P=0.01)$. Almost half of pharmacists in both regions were willing to conduct visual inspection of the genitals, but only eight pharmacists in urban regions and none from rural regions were willing to conduct a physical examination of the genitals $(P=0.05)$. Most pharmacists were not willing to perform syphilis serology.

Familiarity either with the STI syndromic management approach or with the Department of Health syndromic STI management guidelines was limited among pharmacists from both regions. When presented with three clinical STI scenarios, most of the pharmacists responded that they would refer the patient to a doctor and would provide counseling on HIV risk reduction. However, when compared with pharmacists in the urban regions, a significantly smaller proportion of rural pharmacists would counsel these patients $(P=0.01)$. Although few pharmacists would provide medication for male genital ulcer disease, many would treat male urethral discharge $(58 \%$ urban pharmacists and $40 \%$ of rural pharmacists). More than $70 \%$ of pharmacists from both regions indicated that they would provide medication for vaginal discharge. Importantly, of those who would provide medication, very few were able to select the correct syndromic treatment.

In bivariate logistic regression analyses of willingness of pharmacists to provide syndromic STI treatment, statistically significant variables were male gender of the pharmacist, having received prior requests from clientele for dispensing treatment for male genital ulcers without a prescription, knowledge of the existence of syndromic STI treatment protocols, and affirmative responses to the clinical scenarios in which the pharmacist would counsel the patient regarding the link between HIV and STI.

In multivariate analysis, three independent associations with pharmacists' willingness to treat STI were identified. Pharmacists were more likely to be willing to treat STI if they were aware of the HIV/STI link (odds ratio [OR]: 13.8; 95\% CI: 2.7, 70.7) and if they had used they had used the syndromic STI management approach in the past (OR: 11.1; 95\% CI: 1.1, 108.6). Of note, the effects of knowledge of the STI/HIV link and previous use of syndromic STI treatment were not additive (interaction term OR: 0.07; 95\% CI: 0.01, 0.97). Women pharmacists were significantly less likely to treat STI (OR 0.23 ; $95 \% \mathrm{CI}$ : $0.06,0.87)$. 
Pharmacists reported substantial obstacles to conducting STI treatment. The most frequently cited barriers were the current legal restrictions to pharmacists prescribing medication (97.6\%), lack of skills in phlebotomy (97.6\%), and conducting visual (83.5\%) and physical examinations (91.7\%).

\begin{tabular}{|c|c|c|c|}
\hline Characteristic & $\begin{array}{l}\text { Urban } \\
\text { (Cape Metropole) }\end{array}$ & $\begin{array}{c}\text { Rural } \\
\text { (Outside Metropole) }\end{array}$ & $P$ value \\
\hline $\begin{array}{l}\text { Total } \\
\text { Gender of head pharnacist }\end{array}$ & 65 & 30 & \\
\hline \multicolumn{4}{|l|}{ Gender of head pharmacist" } \\
\hline Number (\%) male & $47(85.5)$ & $16(53.3)$ & \multirow[t]{2}{*}{$<0.001$} \\
\hline Number (\%) fernale & 8 (14.5) & $14(46.7)$ & \\
\hline \multicolumn{4}{|l|}{ Pharnacy hours } \\
\hline Medlan (range) hours open per day & $10(8-14)$ & $10(7.5-13)$ & 0.35 \\
\hline Number $(\%)$ open on Saturday & $55(100)$ & $30(100)$ & $\mathrm{NA}^{2}$ \\
\hline Number $(\%)$ open on Sunday & $28(50.91)$ & $16(53.3)$ & 0.83 \\
\hline Nurse enployed at pharmacy & $17(30.9)$ & $14(46.7)$ & 0.15 \\
\hline \multicolumn{4}{|l|}{ Socioeconomic status of population served } \\
\hline Number (\%) upper & $12(21.8)$ & $6(20)$ & \multirow{3}{*}{0.64} \\
\hline Number $(\%)$ lower & $20(36.4)$ & $14(46.7)$ & \\
\hline Number $(\%)$ mixed & $23(41.8)$ & $10(33.3)$ & \\
\hline \multicolumn{4}{|c|}{ Medlan daly number (range) of cllents who requested therapy } \\
\hline \multirow{2}{*}{\multicolumn{4}{|c|}{$\begin{array}{l}\text { Medlan number (range) of cllents who requested STI therapy } \\
\text { from pharmacist without a prescription in past } 4 \text { weeks }\end{array}$}} \\
\hline & & & \\
\hline Vaginal dlscharge & $20(1-98)$ & $6(0-60)$ & $<0.001$ \\
\hline Fernale genital ulcer & $0(0-20)$ & $0(0-2)$ & 0.2 \\
\hline Male geniltal ulcer & $1(0-30)$ & $0(0-8)$ & 0.1 \\
\hline Male penle dlscharge & $5(0-96)$ & $3(0-30)$ & 0.04 \\
\hline
\end{tabular}

'The head pharmacist was defined as the owner or manager of the pharmacy.

NA = not applicable; STI = sexually transmitted infection.

\section{Discussion}

Our study confirms anecdotal reports that a high volume of possible STI is seen in community pharmacies with requests for medication without prescriptions. Furthermore, many community pharmacists recognize the relationship between HIV infection and other STIs, regard their role in treating STI as underutilized, and are willing to provide STI treatment. However, pharmacists cited current legal restrictions and lack of skills as significant barriers to providing such treatment. In addition, the pharmacists surveyed demonstrated a poor understanding of correct syndromic STI treatment protocols. Similarly, poor understanding of recommended treatment protocols was found in an intervention study in Peru in which pharmacies were randomized into those that were provided with syndromic treatment algorithms and those that did not receive training. When presented with research staff posing as patients, less than $16 \%$ of the pharmacists in the intervention or control groups provided the recommended treatment. However, the study in Peru did not assess pharmacists' willingness to receive training and provide treatment; in fact, only $56 \%$ of the pharmacists who were offered training received any. In our study, approximately half of the pharmacists surveyed were willing to provide treatment. Therefore, methods to increase pharmacists' participation in STI syndromic training programs need to be developed.

Contrary to our expectations, the pharmacist's perception of the need for STI services to be provided in pharmacies was not associated with their willingness to treat STIs; rather, the strongest predictor of their willingness to provide treatment was their knowledge of the relationship between STI and HIV infection. It is worth noting that of the steps included in syndromic treatment of STIs, pharmacists were generally willing to conduct all except examination and phlebotomy. Thus, if pharmacists were to be trained to provide STI treatment, efforts to overcome this barrier through specific education and training would need to be implemented. However, because of the difficulty associated 
with training pharmacists in conducting physical examinations, phlebotomy, and biosafety, modified protocols in which the pharmacist relied on the patients' clinical history, possibly with visual inspection of the genital region, would need to be developed and evaluated. Furthermore, lifting of the current legal restrictions that prevent pharmacists from prescribing medication is necessary.

This study has several limitations. We sampled only 90 pharmacies; however, this does represent $22 \%$ of the target population. In addition, response bias may have been introduced, given the legal context of some of the practices of pharmacists. Some of the information collected may not be accurate, particularly the data concerning client characteristics (e.g., income level), for which we relied on pharmacists' observations and impressions. In addition, we did not ascertain information that would allow us to assess an association between the pharmacists' willingness to provide treatment and the various monetary benefits associated with syndromic treatment. Provision of certain medications may have a greater financial benefit to the pharmacists, which might increase willingness to provide treatment. We requested information on the number of clients requesting treatment without a prescription, but did not ascertain the overall number of patrons seen at the pharmacies because many people purchase items unrelated to prescription medications; this limited our ability to determine the proportion of clients who attended the pharmacies who requested treatment without a prescription Also, it should be noted that the reported average STI vaginal discharge caseload is probably an overestimation, given that every vaginal discharge, regardless of etiology, was considered to be an STI. However, because these factors did not contribute significantly to our results, it is unlikely that they invalidate our findings.

Despite these limitations, our study points to a potentially powerful intervention to increase STI treatment coverage and hence reduce HIV transmission. Licensed pharmacists in South Africa have attained a high level of education in the health field, and it is likely that they can be trained to provide appropriate treatment and referrals for common STI syndromes. Factors such as the volume of patients requesting STI treatment, the availability of other local health services, and pharmacists' willingness to be trained could be used to target those pharmacists where training and provision of treatment would be most efficient. We believe that the training and evaluation of pharmacists to provide syndromic treatment of STIs may help in the control of STI and contribute to a reduction in HIV transmission. 
TABLE 2. Knowledge, Attitudes, and Practices Pegarding Sexualy Transmitted infection (ST) Management Among Pharmacists in the Western Cape, South Africa $(\mathrm{N}=85)$

\begin{tabular}{|c|c|c|c|}
\hline Varlable & $\begin{array}{c}\text { Urban } \\
(n=55)\end{array}$ & $\begin{array}{c}\text { Rural } \\
(\mathrm{n}=30)\end{array}$ & $P$ value \\
\hline \multicolumn{4}{|l|}{ Opinion of current role of pharmacists In STI management } \\
\hline Overutllized & $0(0)$ & $0(0)$ & \\
\hline Satisfactory & $12(21.8)$ & $8(26.7)$ & \\
\hline Underutllized & $41(74.6)$ & 22 (73.3) & 0.5 \\
\hline Not sure & $2(3.6)$ & $0(0)$ & \\
\hline \multicolumn{4}{|l|}{ Somewhat or strongy agree that there is a need for } \\
\hline \multirow{2}{*}{\multicolumn{4}{|c|}{ WIIIngness to perform IndMidual STI management steps }} \\
\hline & & & \\
\hline History taking & $55(100)$ & $28(93.3)$ & 0.12 \\
\hline Visual examination of genitals & $27(49.1)$ & $14(46.7)$ & 0.83 \\
\hline Physical examination of genitals & $8(14.6)$ & $0(0)$ & 0.06 \\
\hline Prescribing & $55(100)$ & $26(66.7)$ & 0.01 \\
\hline Counseling for HIV/STI prevention & $55(100)$ & 28 (93.3) & 0.12 \\
\hline Draw blood for syphlls & 17 (30.9) & $8(26.7)$ & 0.68 \\
\hline Partner-notification counseling & $55(100)^{\prime}$ & $28(93.3)$ & 0.12 \\
\hline Follow-up & $55(100)$ & $28(93.3)$ & 0.12 \\
\hline Overall willingness to provide STI treatment ${ }^{4}$ & $29(52.7)$ & $14(46.7)$ & 0.59 \\
\hline \multicolumn{4}{|l|}{ Famillarity with and use of syndromic STI treatment } \\
\hline Heard of and use syndromic STI treatment & $0(0)$ & $2(6.7)$ & \\
\hline Heard of but do not use syndromic STI treatment & $12(21.8)$ & $10(33.3)$ & 0.06 \\
\hline Never heard of syndromic STI treatment & $43(78.2)$ & $18(60)$ & \\
\hline \multicolumn{4}{|l|}{$\begin{array}{l}\text { Famillarity with and use of the Department of Health } \\
\text { syndromic STI management gulde }\end{array}$} \\
\hline Heard of and use the treatment gulde & $2(3.6)$ & $4(13.3)$ & \\
\hline Heard of but do not use the treaiment gulde & $12(21.8)$ & $8(26.7)$ & 0.20 \\
\hline Never heard of the treatment gulde & $41(74.6)$ & $18(60.0)$ & \\
\hline \multicolumn{4}{|l|}{$\begin{array}{l}\text { Management approach to hypothetical male pattent with } \\
\text { urethral discharge }\end{array}$} \\
\hline Would refer to doctor & 37 (67.3) & $22(73.3)$ & 0.56 \\
\hline Would counsel regarding HIV risk reduction & 47 (85.5) & $18(60.0)$ & 0.01 \\
\hline Would provide medication & 32 (58.2) & $12(40.0)$ & 0.11 \\
\hline \multicolumn{4}{|l|}{ Correctness of medication ( $n=44$ ) } \\
\hline Selected effectlve combination of theraples & 4 (12.5) & $2(16.7)$ & 0.7 \\
\hline Selected partially effectlve theraples & $24(75)$ & $6(50)$ & 0.0000 \\
\hline \multicolumn{4}{|c|}{$\begin{array}{l}\text { Management approach to hypothetical male pattent with a } \\
\text { garital ulcer }\end{array}$} \\
\hline Would refer to doctor & $39(70.9)$ & $26(66.7)$ & 0.1 \\
\hline Would counsel regarding the HIV risk reduction & 47 (85.6) & $18(60.0)$ & 0.01 \\
\hline Would provide medication & 26 (47.3́) & $6(20.0)$ & 0.01 \\
\hline \multicolumn{4}{|l|}{ Correctness of medication ( $n=32$ ) } \\
\hline Selected effective combination of theraples & $2(7.7)$ & $0(0)$ & 0.81 \\
\hline Selected partlally effectlve theraples & 6 (23.1) & $2(33.3)$ & 0.0001 \\
\hline \multicolumn{4}{|c|}{$\begin{array}{l}\text { Management approach to hypothetical patient with vaginal } \\
\text { discharge }\end{array}$} \\
\hline Would refer to doctor & $30(60.0)$ & $16(53.3)$ & 0.91 \\
\hline Would counsel the HIV risk reduction & 47 (85.6) & $24(60)$ & 0.73 \\
\hline Would provide medication & $39(70.9)$ & $22(73.3)$ & 0.81 \\
\hline \multicolumn{4}{|l|}{ Correctness of medication ( $n=61)$} \\
\hline Selected effectlve combination of theraples & 0 & 0 & \\
\hline Selected partially effective theraples & $23(59.0)$ & $4(18.2)$ & 0.0000 \\
\hline
\end{tabular}




\section{References}

1. Joint United Nations Programme on HIV/AIDS (UNAIDS)/WHO. UNAIDS epidemic update 2002. Geneva: UNAIDS, 2002

2. Department of Health. HIV/AIDS/STI strategic plan for South Africa 2000 -2005. Pretoria: Ministry of Health, February 2000.

3. Flemming DT, Wasserheit JN. From epidemiological synergy to public health policy and practice: the contribution of other sexually transmitted diseases to sexual transmission of HIV infection. Sex Transm Infect 1999; 75:3-17.

4. Grosskurth H, Mosha F, Todd J, et al. Impact of improved treatment of sexually transmitted diseases on HIV infection in rural Tanzania: randomised controlled trial. Lancet 1995; 346:530 - 536.

5. WHO/HIV-AIDS/2001.01. Guidelines for the management of STI. Geneva: World Health Organization, 2001.

6. Ballard R, Htun Y, Fehler G, Neilsen G. The Diagnosis and Management of Sexually Transmitted Infections in Southern Africa, 3rd ed. Johannesburg: South African Institute for Medical Research, 2000.

7. Pick W, Fisher B, Kowo H. Measuring the quality of care in South African clinics and hospitals. Technical report. In: Ntuli A, ed.

South African Health Review. Durban: Health Systems Trust, 1998.

1. Dartnall E, Schneider H, Hlatshwayo Z, Clews F. STI Management in Private Sector: A National Evaluation. Johannesburg: Centre for Health Policy, 1997.

2. Garcia PJ, Gotuzzo E, Hughes JP, Holmes KK. Syndromic management of STIs in pharmacies: evaluation and randomised intervention trial. Sex Transm Infect 1998; 74(Suppl 1):S153-S158.

3. Adu-Sarkodie Y, Steiner MJ, Attafuah J, Tweedy K. Syndromic management of urethral discharge in Ghanaian pharmacies. Sex Transm

Infect 2000; 76:439-442. 